\title{
Nucleon Matrix Elements of the Antisymmetric Quark Tensor
}

\author{
Martin Hoferichter, ${ }^{1}$ Bastian Kubis, ${ }^{2}$ Jacobo Ruiz de Elvira, ${ }^{3}$ and Peter Stoffer ${ }^{4}$ \\ ${ }^{1}$ Institute for Nuclear Theory, University of Washington, Seattle, Washington 98195-1550, USA \\ ${ }^{2}$ Helmholtz-Institut für Strahlen- und Kernphysik (Theorie) and Bethe Center for Theoretical Physics, \\ Universität Bonn, 53115 Bonn, Germany \\ ${ }^{3}$ Albert Einstein Center for Fundamental Physics, Institute for Theoretical Physics, University of Bern, \\ Sidlerstrasse 5, 3012 Bern, Switzerland \\ ${ }^{4}$ Department of Physics, University of California at San Diego, La Jolla, California 92093, USA
}

(Received 28 November 2018; revised manuscript received 29 January 2019; published 29 March 2019)

\begin{abstract}
If physics beyond the standard model enters well above the electroweak scale, its low-energy effects are described by standard model effective field theory. Already, at dimension 6, many operators involve the antisymmetric quark tensor $\bar{q} \sigma^{\mu \nu} q$, whose matrix elements are difficult to constrain from experiment, Ward identities, or low-energy theorems, in contrast to the corresponding vector and axial-vector or even scalar and pseudoscalar currents. However, with normalizations determined from lattice QCD, analyticity and unitarity often allow one to predict the momentum dependence in a large kinematic range. Starting from recent results in the meson sector, we extend this method to the nucleon case and, in combination with pole dominance, provide a comprehensive assessment of the current status of the nucleon form factors of the quark tensor.
\end{abstract}

DOI: 10.1103/PhysRevLett.122.122001

Introduction.-When the standard model (SM) is considered an effective low-energy theory, physics beyond the SM (BSM) can be encoded in higher-dimensional operators that supplement the SM Lagrangian but still respect the $\mathrm{SU}(3) \times \mathrm{SU}(2)_{L} \times \mathrm{U}(1)$ gauge symmetries. At dimension 5 , only a single such operator exists, the lepton-numberviolating Weinberg operator [1], but at dimension 6 , a host of new terms become possible [2,3]. Among these are operators that involve quark currents $\bar{q}_{f} \Gamma q_{i}$, with possible Dirac structures $\Gamma \in\left\{\mathbb{1}, \gamma_{5}, \gamma^{\mu}, \gamma^{\mu} \gamma_{5}, \sigma^{\mu \nu}\right\}$. For the calculation of low-energy observables, the matrix elements of these operators are often crucial input quantities, both in mesonic and baryonic systems. However, due to the absence of scalar, pseudoscalar, or tensor probes in the SM, only the matrix elements of vector and axial-vector quark currents can be directly taken from experiment.

Scalar and pseudoscalar operators are related to vector and axial-vector ones by Ward identities [4-6]

$$
\begin{aligned}
\partial_{\mu}\left(\bar{q}_{f} \gamma^{\mu} q_{i}\right) & =i\left(m_{f}-m_{i}\right) \bar{q}_{f} q_{i}, \\
\partial_{\mu}\left(\bar{q}_{f} \gamma^{\mu} \gamma_{5} q_{i}\right) & =i\left(m_{f}+m_{i}\right) \bar{q}_{f} \gamma_{5} q_{i},
\end{aligned}
$$

known as the conservation of the vector current and partial conservation of the axial current, which sometimes imply

Published by the American Physical Society under the terms of the Creative Commons Attribution 4.0 International license. Further distribution of this work must maintain attribution to the author(s) and the published article's title, journal citation, and DOI. Funded by SCOAP . relations among matrix elements, e.g., in the context of nuclear $\beta$ decay [7]. Similarly, the chiral symmetry of QCD provides constraints in the form of low-energy theorems, corrections to which can be systematically studied in chiral perturbation theory [8-10], e.g., the Callan-Treiman lowenergy theorem for $K_{\ell 3}$ and $K_{\ell 4}$ form factors [11-16] or the Cheng-Dashen low-energy theorem for the pion-nucleon $\sigma$-term [17-20]. In contrast, for the tensor current, even in the meson sector, unknown low-energy constants appear already at leading order [21], so that, in general, matrix elements of the quark tensor current are difficult to constrain from experiment, both directly and indirectly, with the only indirect connection via moments of parton distribution functions [22-27]. Accordingly, these matrix elements are critical input quantities for BSM searches in a variety of processes, including kaon $[28,29]$ and $\tau$ decays [30-32], dark matter searches [33-37], $\mu \rightarrow e$ conversion in nuclei [38-41], and electric dipole moments $[42,43]$.

In recent years, precise calculations of the tensor charges from lattice QCD have become available both for mesons $[44,45]$ and the nucleon [46-49], which thus determine certain form factor normalizations. Depending on the application, also the momentum dependence becomes relevant, but to constrain this behavior additional information is available from analyticity and unitarity of the form factors. In fact, as long as a single intermediate state dominates the unitarity relation, an exact representation can be given in terms of amplitudes that, at least in principle, are accessible in experiment as well as the normalization as determined from lattice QCD. Physically, such relations 


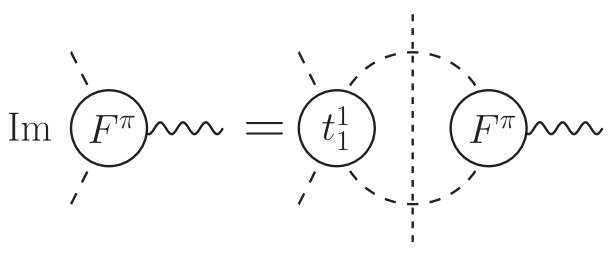

FIG. 1. Elastic unitarity relation for the pion form factors $F^{\pi}=\left\{F_{\pi}^{V}, B_{T}^{\pi, q}\right\}$. The dashed lines denote pions, the wiggly lines the external current, and the short-dashed line indicates that the intermediate-state particles are to be taken on shell.

come about because a vector resonance can be described equivalently by a vector or an antisymmetric tensor field $[50,51]$, in such a way that the same hadronic resonances appear in both form factors. This strategy has been used recently in the context of $\tau$ decays [31,32].

In this Letter, we focus on the nucleon matrix elements. First, we consider $\pi \pi$ intermediate states and derive the corresponding unitarity relation, in analogy to the electromagnetic form factors [52-57]. In combination with a narrow $\omega$ resonance, this determines all $2 \pi$ and $3 \pi$ contributions corresponding to $J^{P C}=1^{--}$quantum numbers. As first pointed out in [58], there are also contributions from the $1^{+-}$channel, dominated by the $h_{1}(1170)$ and $b_{1}(1235)$ resonances for isospin $I=0$ and $I=1$, which mainly couple to $3 \pi$ and $4 \pi$ intermediate states, respectively. Combining all information from lattice-QCD tensor charges, analyticity, unitarity, and pole dominance, we provide a complete description of the nucleon tensor form factors below $\sqrt{|t|} \lesssim 1 \mathrm{GeV}$.

Meson form factors.-The standard decomposition of the pion matrix element of the electromagnetic current

$$
j_{\mathrm{em}}^{\mu}=\sum_{q=u, d, s} Q_{q} \bar{q} \gamma^{\mu} q, \quad Q=\frac{1}{3} \operatorname{diag}(2,-1,-1)
$$

reads

$\left\langle\pi^{+}\left(p^{\prime}\right)\left|j_{\mathrm{em}}^{\mu}\right| \pi^{+}(p)\right\rangle=\left(p+p^{\prime}\right)^{\mu} F_{\pi}^{V}(t), \quad t=\left(p^{\prime}-p\right)^{2}$,

where charge conservation determines the normalization of the form factor $F_{\pi}^{V}(0)=1$. Elastic unitarity gives the imaginary part from $\pi \pi$ intermediate states (see Fig. 1),

$$
\operatorname{Im} F_{\pi}^{V}(t)=\sigma_{\pi}(t)\left[t_{1}^{1}(t)\right]^{*} F_{\pi}^{V}(t)
$$

where $\sigma_{\pi}(t)=\sqrt{1-4 M_{\pi}^{2} / t}$ and $t_{1}^{1}(t)$ is the $P$-wave $\pi \pi$ partial-wave amplitude related to the phase shift $\delta_{1}^{1}(t)$ according to

$$
t_{1}^{1}(t)=\frac{1}{\sigma_{\pi}(t)} e^{i \delta_{1}^{1}(t)} \sin \delta_{1}^{1}(t)
$$

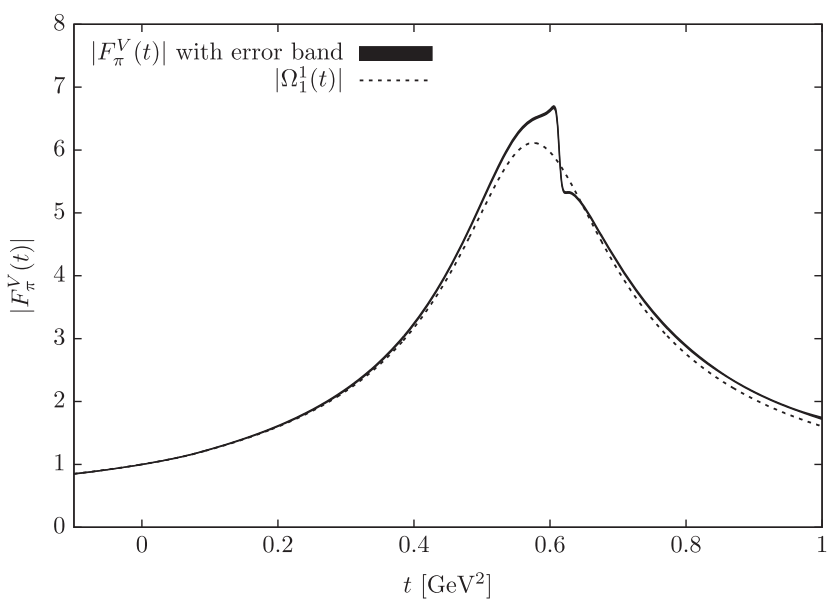

FIG. 2. Comparison of the Omnès factor $\Omega_{1}^{1}$ to the full result for $F_{\pi}^{V}[70]$.

Equation (4) is a manifestation of Watson's final-state theorem [59] that equates the phase of the form factor with $\delta_{1}^{1}(t)$, leading to a representation in terms of the Omnès function [60]

$F_{\pi}^{V}(t)=\Omega_{1}^{1}(t), \quad \Omega_{1}^{1}(t)=\exp \left(\frac{t}{\pi} \int_{4 M_{\pi}^{2}}^{\infty} d t^{\prime} \frac{\delta_{1}^{1}\left(t^{\prime}\right)}{t^{\prime}\left(t^{\prime}-t\right)}\right)$.

In practice, this representation does not fully capture all properties of $F_{\pi}^{V}(t)$ : corrections arise from inelastic states such as $3 \pi$ in the vicinity of the $\omega(782)$ resonance (isospin violating) as well as $4 \pi$ above $1 \mathrm{GeV}$ (isospin conserving). These effects can be accounted for by suitable extensions of the Omnès representation (see [61-70]), but due to the dominance of the $\rho(770)$, the Omnès factor provides the bulk of the contribution (see Fig. 2). In particular, the lowenergy parameters are well reproduced, e.g., the radius

$$
\left\langle\left(r_{\pi}^{V}\right)^{2}\right\rangle=\left.6 \frac{d F_{\pi}^{V}(t)}{d t}\right|_{t=0}=0.419 \mathrm{fm}^{2}
$$

differs from the full result [70]

$$
\left\langle\left(r_{\pi}^{V}\right)^{2}\right\rangle=0.429(4) \mathrm{fm}^{2}
$$

by about $2 \%$, where the increase with respect to (7) comes from inelastic effects.

For the decomposition of the pion matrix element of the tensor current, we take $[45,71]$

$$
\left\langle\pi^{+}\left(p^{\prime}\right)\left|\bar{q} \sigma^{\mu \nu} q\right| \pi^{+}(p)\right\rangle=\frac{i}{M_{\pi}}\left(p^{\prime \mu} p^{\nu}-p^{\mu} p^{\prime \nu}\right) B_{T}^{\pi, q}(t) .
$$

The crucial observation is that elastic unitarity produces exactly the same result as in (4),

$$
\operatorname{Im} B_{T}^{\pi, q}(t)=\sigma_{\pi}(t)\left[t_{1}^{1}(t)\right]^{*} B_{T}^{\pi, q}(t),
$$


so that up to inelastic corrections we may write

$$
B_{T}^{\pi, q}(t)=B_{T}^{\pi, q}(0) F_{\pi}^{V}(t) .
$$

In principle, analyticity and unitarity alone would allow for an arbitrary polynomial multiplying the Omnès factor, but in the same way as for the vector form factor constraints from perturbative QCD [72] should lead to an asymptotic $1 / t$ behavior. With the normalization determined from lattice QCD [45] (all tensor matrix elements are quoted at an $\overline{\mathrm{MS}}$ scale $\mu=2 \mathrm{GeV}$ ),

$$
B_{T}^{\pi, u}(0)=-B_{T}^{\pi, d}(0)=0.195(10),
$$

this essentially determines the tensor form factor throughout $\sqrt{t} \lesssim 1 \mathrm{GeV}$ except for close to the $\omega$ resonance. In particular, we obtain for the radius

$$
\left\langle\left(r_{T}^{\pi, u}\right)^{2}\right\rangle=\left.\frac{6}{B_{T}^{\pi, u}(0)} \frac{d B_{T}^{\pi, u}(t)}{d t}\right|_{t=0}=0.43(1) \mathrm{fm}^{2},
$$

where we have adopted the central value from (8), assuming that inelastic effects modify $\left\langle\left(r_{T}^{\pi, u}\right)^{2}\right\rangle$ in a similar way as $\left\langle\left(r_{\pi}^{V}\right)^{2}\right\rangle$, and the error is motivated by the corresponding difference between (8) and (7). Phenomenologically, these relations work because of the dominance of the $\rho(770)$.

A similar argument applies to the flavor-nondiagonal current $\bar{s} \sigma^{\mu \nu} u$, whose $\pi K$ form factor

$$
\left\langle\overline{K^{0}}\left(p^{\prime}\right)\left|\bar{s} \sigma^{\mu \nu} u\right| \pi^{+}(p)\right\rangle=\frac{i}{M_{K}}\left(p^{\prime \mu} p^{\nu}-p^{\mu} p^{\prime \nu}\right) B_{T}^{\pi K}(t)
$$

is related by elastic unitarity to the $K_{\ell 3}$ form factor $f_{+}(t)[29]$

$$
B_{T}^{\pi K}(t)=B_{T}^{\pi K}(0) \frac{f_{+}(t)}{f_{+}(0)},
$$

and inelastic corrections are suppressed with respect to the $K^{*}(892)$ resonance. With normalization $B_{T}^{\pi K}(0)=$ $0.686(25)$ [45] and $d f_{+}(t) / d t=f_{+}(0) \lambda_{+}^{\prime} / M_{\pi}^{2}$ as measured in $K_{\ell 3}$ experiments, $\lambda_{+}^{\prime}=25.2(9) \times 10^{-3}$ [29], this gives

$$
\left\langle\left(r_{T}^{\pi K}\right)^{2}\right\rangle=\left.\frac{6}{B_{T}^{\pi K}(0)} \frac{d B_{T}^{\pi K}(t)}{d t}\right|_{t=0}=0.30(2) \mathrm{fm}^{2},
$$

where the uncertainty derives from the comparison of the physical $\lambda_{+}^{\prime}$ with the derivative of the $\pi K$ Omnès factor as an estimate of the impact of inelastic effects.

Finally, we note that the lattice normalizations determine some of the low-energy constants $\Lambda_{i}$ from [21]. The $\pi \pi$ result (12) implies $\Lambda_{2}=F_{\pi}^{2} B_{T}^{\pi, u}(0) /\left(2 M_{\pi}\right)=$ 6.0(3) MeV [73,74], in good agreement with the SU(3)related version $\Lambda_{2}=F_{\pi}^{2} B_{T}^{\pi K}(0) /\left(2 M_{K}\right)=5.9(2) \mathrm{MeV}$.

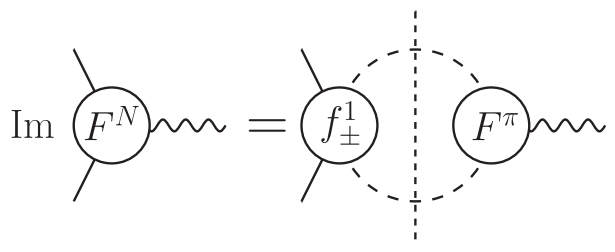

FIG. 3. Elastic unitarity relation for the isovector nucleon form factors $F^{N}=\left\{F_{i}, F_{i, T}^{q}\right\}$ and accordingly $F^{\pi}=\left\{F_{\pi}^{V}, B_{T}^{\pi, q}\right\}$. The solid lines denote nucleons, otherwise notation as in Fig. 1.

Further, by assuming $\rho$ pole dominance, we obtain the relation (see also [73])

$$
\Lambda_{1}=-\frac{M_{\rho}^{2} B_{T}^{\pi, u}(0)}{4 g_{\rho \pi \pi} g_{\rho \gamma} M_{\pi}}=-7.1(7) \mathrm{MeV}
$$

in terms of the $\rho \pi \pi$ and $\rho \gamma$ coupling constants [75-77], and we have attached a $10 \%$ uncertainty as suggested by the analog estimate for the charge radius $\left\langle\left(r_{\pi}^{V, \rho}\right)^{2}\right\rangle=$ $6 / M_{\rho}^{2}=0.39 \mathrm{fm}^{2}$.

Nucleon form factors.-The nucleon transition matrix element of the electromagnetic current operator has the standard form

$\left\langle N\left(p^{\prime}\right)\left|j_{\mathrm{em}}^{\mu}\right| N(p)\right\rangle=\bar{u}\left(p^{\prime}\right)\left[\gamma^{\mu} F_{1}(t)+\frac{i \sigma^{\mu \nu} q_{\nu}}{2 m_{N}} F_{2}(t)\right] u(p)$,

where $q=p^{\prime}-p$. For the isovector combinations $F_{i}^{v}=$ $\left(F_{i}^{p}-F_{i}^{n}\right) / 2, \pi \pi$ intermediate states again dominate the unitarity relation, in such a way that the strategy from the mesonic system generalizes in a straightforward way to the nucleon case (see Fig. 3). The main difference, however, concerns the fact that on the right-hand side of the unitarity relation both the scattering amplitude, $\pi \pi \rightarrow N \bar{N}$ in this case, and the pion form factor enter as input. Following the notation from $[68,78]$, we have $[52,53,79]$

$\operatorname{Im} F_{1}^{v}(t)=\frac{q_{t}^{3}}{2 \sqrt{t}}\left[F_{\pi}^{V}(t)\right]^{*}\left(\frac{2 m_{N}}{p_{t}^{2}} \Gamma^{1}(t)+\sqrt{2} f_{-}^{1}(t)\right)$,

$\operatorname{Im} F_{2}^{v}(t)=-\frac{m_{N} q_{t}^{3}}{p_{t}^{2} \sqrt{t}}\left[F_{\pi}^{V}(t)\right]^{*} \Gamma^{1}(t)$,

where $f_{ \pm}^{1}(t)$ are the $P$-wave partial waves for $\pi \pi \rightarrow N \bar{N}$,

$$
\Gamma^{1}(t)=\frac{m_{N}}{\sqrt{2}} f_{-}^{1}(t)-f_{+}^{1}(t),
$$

and $p_{t}=\sqrt{t / 4-m_{N}^{2}}, q_{t}=\sqrt{t / 4-M_{\pi}^{2}}$.

The matrix elements of the tensor current decompose according to $[80,81]$ 


$$
\begin{aligned}
\left\langle N\left(p^{\prime}\right)\left|\bar{q} \sigma^{\mu \nu} q\right| N(p)\right\rangle & \\
= & \bar{u}\left(p^{\prime}\right)\left[\sigma^{\mu \nu} F_{1, T}^{q}(t)+\frac{i}{m_{N}}\left(\gamma^{\mu} q^{\nu}-\gamma^{\nu} q^{\mu}\right) F_{2, T}^{q}(t)\right. \\
& \left.+\frac{i}{m_{N}^{2}}\left(P^{\mu} q^{\nu}-P^{\nu} q^{\mu}\right) F_{3, T}^{q}(t)\right] u(p) \\
= & \bar{u}\left(p^{\prime}\right)\left[\sigma^{\mu \nu} F_{1, T}^{q}(t)+\frac{i}{m_{N}}\left(\gamma^{\mu} q^{\nu}-\gamma^{\nu} q^{\mu}\right) \hat{F}_{2, T}^{q}(t)\right. \\
& \left.+\frac{1}{m_{N}^{2}}\left(\sigma^{\mu \alpha} q^{\nu}-\sigma^{\nu \alpha} q^{\mu}\right) q_{\alpha} F_{3, T}^{q}(t)\right] u(p),
\end{aligned}
$$

where $P=p+p^{\prime}$ and $\hat{F}_{2, T}^{q}(t)=F_{2, T}^{q}(t)+2 F_{3, T}^{q}(t)$. As expected, the evaluation of the unitarity relation of the tensor form factors from $\pi \pi$ intermediate states produces a result that only depends on the $P$-wave amplitudes. We find

$$
\begin{aligned}
\operatorname{Im} F_{1, T}^{q, v}(t) & =0 \\
\operatorname{Im} F_{2, T}^{q, v}(t) & =-\frac{m_{N} q_{t}^{3}}{2 M_{\pi} \sqrt{2 t}}\left[B_{T}^{\pi, q}(t)\right]^{*} f_{-}^{1}(t), \\
\operatorname{Im} F_{3, T}^{q, v}(t) & =-\frac{m_{N}^{2} q_{t}^{3}}{4 M_{\pi} p_{t}^{2} \sqrt{t}}\left[B_{T}^{\pi, q}(t)\right]^{*} \Gamma^{1}(t) .
\end{aligned}
$$

Using (19) and (11), this leads to the identification

$$
\begin{aligned}
& F_{1, T}^{q, v}(t)=0, \\
& F_{2, T}^{q, v}(t)=-\frac{m_{N}}{2 M_{\pi}} B_{T}^{\pi, q}(0)\left[F_{1}^{v}(t)+F_{2}^{v}(t)\right], \\
& F_{3, T}^{q, v}(t)=\frac{m_{N}}{4 M_{\pi}} B_{T}^{\pi, q}(0) F_{2}^{v}(t),
\end{aligned}
$$

for the $I=1, J^{P C}=1^{--}$contribution, valid up to inelastic corrections. In particular, vector intermediate states do not contribute to $F_{1, T}^{q, v}$. As a first check on these relations we consider the tensor anomalous magnetic moments $\kappa_{T}^{q}=-2 \hat{F}_{2, T}^{q}(0)$ and compare to lattice QCD [82]

$$
\kappa_{T}^{u, p} \approx 3.0, \quad \kappa_{T}^{d, p} \approx 1.9,
$$

in reasonable agreement with (23)

$$
1.1 \approx \kappa_{T}^{u, p}-\kappa_{T}^{u, n}=\frac{m_{N}}{M_{\pi}} B_{T}^{\pi, u}(0)=1.31(7),
$$

where we have assumed isospin symmetry $\kappa_{T}^{u, n}=\kappa_{T}^{d, p}$, etc. In fact, the identification (23) is precisely what one would have obtained assuming a narrow resonance to describe the $\rho$ : using the Lagrangian from [83], we find

$$
F_{1}^{v, \rho}(t)=\frac{1}{2} D_{\rho}(t), \quad F_{2}^{v, \rho}(t)=\frac{\kappa_{\rho}}{2} D_{\rho}(t),
$$

with $D_{\rho}(t)=M_{\rho}^{2} /\left(M_{\rho}^{2}-t\right)$, where $g_{\rho}=g_{\rho \gamma}$ has been assumed to ensure the correct normalization of $F_{1}^{v}$, and $\kappa_{\rho}$ parametrizes the magnetic-moment coupling of the $\rho$ in the conventions of [83]. Moreover, $\rho$ pole dominance gives for the ratio of tensor and vector coupling constants

$$
\frac{F_{\rho}^{T}}{F_{\rho}}=\frac{B_{T}^{\pi, u}(0)}{2} \frac{M_{\rho}}{M_{\pi}}=0.54(3),
$$

in reasonable agreement with the most recent result from lattice QCD $F_{\rho}^{T} / F_{\rho}=0.629(8)$ [84] and not far from the expectation $1 / \sqrt{2}$ from large $N_{c}$ [85]. Accordingly, the calculation of the nucleon tensor form factors then reproduces (23), in such a way that the dispersive derivation in terms of the spectral functions should be considered a more rigorous justification that does not rely on a narrowresonance assumption, only on the dominance of elastic intermediate states.

Beyond the isovector combination, these arguments for (23) suggest to estimate the isoscalar $1^{--}$contributions in a similar way based on a narrow $\omega$ as a description of the $3 \pi$ channel,

$$
F_{1}^{s, \omega}(t)=\frac{1}{2} D_{\omega}(t), \quad F_{2}^{s, \omega}(t)=\frac{\kappa_{\omega}}{2} D_{\omega}(t) .
$$

Lattice results for $F_{\phi}^{T} / F_{\phi}$ indicate a deviation from the $\rho$ around 10\% [86-88], which in turn implies that $F_{\omega}^{T} / F_{\omega}$ should be very close as well, given that the $\mathrm{SU}(3)$ corrections are absent, while the small Okubo-ZweigIizuka rule violations only increase by a factor of 2 [89]. In this way, we obtain an isovector analog of (23) up to an overall factor of $g_{\omega \gamma} / g_{\rho \gamma}=3$. Indeed, the comparison to the isoscalar combination of tensor anomalous magnetic moments

$$
4.9 \approx \kappa_{T}^{u, p}+\kappa_{T}^{u, n}=3 \frac{m_{N}}{M_{\pi}} B_{T}^{\pi, u}(0)=3.94(20)
$$

works at a similar level as (25), and both are in reasonable agreement given the exploratory character of the lattice results [82] and the fact that the dispersive derivation relies on an unsubtracted dispersion relation. Note that, even for the channels for which no rigorous spectral functions as in (22) are known, an ansatz for the full momentum dependence with good analytic properties can be made by replacing the narrow-width propagators by a dispersion relation

$\frac{1}{\pi} \int_{t_{\mathrm{thr}}}^{\infty} d t^{\prime} \frac{\operatorname{Im} d_{R}\left(t^{\prime}\right)}{t^{\prime}-t}, \quad d_{R}(t)=\frac{1}{M_{R}^{2}-t-i M_{R} \Gamma_{R}}$,

or variants thereof, e.g., with an energy-dependent width [90-93].

Next, we turn to the contributions from the $C$-odd axials $h_{1}(1170)$ and $b_{1}(1235)[58,94]$. Modifying the Lagrangian 
from [83] by Levi-Civita tensors to account for the different parity, we find that only one structure produces a resonant contribution,

$$
\begin{aligned}
& F_{1, T}^{q, a}(t)=F_{1, T}^{q}(0) D_{R}(t), \quad \hat{F}_{2, T}^{q, a}(t)=0, \\
& F_{3, T}^{q, a}(t)=-F_{1, T}^{q}(0) \frac{m_{N}^{2}}{M_{R}^{2}} D_{R}(t),
\end{aligned}
$$

where $R=h_{1}(1170)$ and $R=b_{1}(1235)$ for the isoscalar and isovector combinations, respectively, and the superscript $a$ indicates the new axial-vector contribution (parity forbids such a coupling for the pseudoscalar mesons). The tensor charges [49] determine the normalizations according to

$F_{1, T}^{u, p}(0)=0.784(28), \quad F_{1, T}^{d, p}(0)=-0.204(11)$.

Taking together vector and axial-vector contributions, we find the representation

$$
\begin{aligned}
F_{1, T}^{q, p}(t)= & \pm \frac{1}{2}\left[F_{1, T}^{u, p}(0)-F_{1, T}^{d, p}(0)\right] D_{b_{1}}(t) \\
& +\frac{1}{2}\left[F_{1, T}^{u, p}(0)+F_{1, T}^{d, p}(0)\right] D_{h_{1}}(t), \\
F_{2, T}^{u, p}(t)= & -\frac{m_{N}}{2 M_{\pi}} B_{T}^{\pi, u}(0)\left[2 G_{M}^{p}(t)+G_{M}^{n}(t)\right]+2 \tilde{F}_{1, T}^{u, p}(t), \\
F_{2, T}^{d, p}(t)= & -\frac{m_{N}}{2 M_{\pi}} B_{T}^{\pi, u}(0)\left[G_{M}^{p}(t)+2 G_{M}^{n}(t)\right]+2 \tilde{F}_{1, T}^{d, p}(t), \\
F_{3, T}^{u, p}(t)= & \frac{m_{N}}{4 M_{\pi}} B_{T}^{\pi, u}(0)\left[2 F_{2}^{p}(t)+F_{2}^{n}(t)\right]-\tilde{F}_{1, T}^{u, p}(t), \\
F_{3, T}^{d, p}(t)= & \frac{m_{N}}{4 M_{\pi}} B_{T}^{\pi, u}(0)\left[F_{2}^{p}(t)+2 F_{2}^{n}(t)\right]-\tilde{F}_{1, T}^{d, p}(t),
\end{aligned}
$$

where \pm corresponds to $q=u, d, G_{M}=F_{1}+F_{2}, \tilde{F}_{1, T}^{q}$ is $F_{1, T}^{q, a}(t)$ multiplied by $m_{N}^{2} / M_{R}^{2}$, and the neutron form factors follow from isospin symmetry. Our final results for normalizations and slopes according to (33) are collected in Table I. The uncertainties are estimated as follows: First, the part of the normalizations of $F_{2, T}^{q}$ and $F_{3, T}^{q}$ derived from the electromagnetic form factors is assigned a $40 \%$ uncertainty, corresponding to the sum-rule violations observed in [68]. Similarly, the isovector sum rules for the electromagnetic radii suggest an accuracy of the derivatives at a level of $10 \%$. In both cases, the isoscalar extension to the $\omega$ (and $\phi$ ) should hold at a similar level. This expectation follows from the fact that the reason for the slow convergence of the isovector sum rules as well as the departure from simple pole dominance for the $\rho$, e.g., compared to the meson form factors discussed above, traces back to the singularity structure of the nucleon Born terms in $\pi \pi \rightarrow N \bar{N}$, but this threshold enhancement is compensated by phase space for intermediate states with higher multiplicity such as $3 \pi$ [95]. In the same way, since the $1^{+-}$resonances mainly couple to the $3 \pi$ and $4 \pi$ channels, pole dominance should again work reasonably well, as suggested by the meson examples
TABLE I. Summary of normalizations and radii or slopes for the meson and nucleon tensor form factors.

\begin{tabular}{lcc}
\hline \hline Form factor & Normalization & Radius $\left(\mathrm{fm}^{2}\right)$ \\
\hline$B_{T}^{\pi, u}=-B_{T}^{\pi, d}$ & $0.195(10)$ & $0.43(1)$ \\
$B_{T}^{\pi K}$ & $0.686(25)$ & $0.30(2)$ \\
\hline
\end{tabular}

\begin{tabular}{lcc}
\hline \hline Form factor & Normalization & Radius $\left(\mathrm{fm}^{2}\right)$ \\
\hline$F_{1, T}^{u, p}$ & $0.784(28)$ & $0.54(11)$ \\
$F_{1, T}^{d, p}$ & $-0.204(11)$ & $-0.11(2)$ \\
$F_{2, T}^{u, p}$ & $-1.5(1.0)$ & $-7.0(8)$ \\
$F_{2, T}^{d, p}$ & $0.5(3)$ & $2.5(3)$ \\
$F_{3, T}^{u, p}$ & $0.1(2)$ & $1.8(2)$ \\
$F_{3, T}^{d, p}$ & $-0.6(3)$ & $-2.1(2)$ \\
$F_{1, T}^{s, N}$ & $-0.0027(16)$ & $-0.0014(9)$ \\
$F_{2, T}^{s, N}$ & $-0.006(3)$ & $-0.005(8)$ \\
$F_{3, T}^{s, N}$ & $0.003(2)$ & $0.001(4)$ \\
\hline \hline
\end{tabular}

we attach a $20 \%$ uncertainty. Indeed, the corresponding slopes agree well with $\dot{F}_{1, T}^{u, p}=0.57(3) \mathrm{GeV}^{-2}$ and $\dot{F}_{1, T}^{d, p}=$ $-0.14(2) \mathrm{GeV}^{-2}$ from lattice QCD [96] (note that these errors are incomplete, e.g., due to disconnected diagrams). In all cases, the uncertainties associated with sum-rule convergence and pole dominance by far outweigh the uncertainties in the nucleon form factors. For definiteness, we take magnetic moments and $\left\langle r_{E}^{2}\right\rangle^{n}=-0.12 \mathrm{fm}^{2}$ from [74] (the latter mainly based on [97,98]), as well as $r_{E}^{p}=$ $0.84 \mathrm{fm}[99,100], r_{M}^{p}=0.87 \mathrm{fm}, r_{M}^{n}=0.89 \mathrm{fm} \mathrm{[101].}$

Finally, we remark that, assuming SU(3) symmetry for the tensor coupling, the strangeness form factors can be estimated in close analogy to (33),

$$
\begin{aligned}
& F_{1, T}^{s, N}(t)=F_{1, T}^{s, N}(0) D_{h_{1}^{s}}(t), \\
& F_{2, T}^{s, N}(t)=-\frac{m_{N}}{2 M_{\pi}} B_{T}^{\pi, u}(0) G_{M}^{s, N}(t)+2 \tilde{F}_{1, T}^{s, N}(t), \\
& F_{3, T}^{s, N}(t)=\frac{m_{N}}{4 M_{\pi}} B_{T}^{\pi, u}(0) F_{2}^{s, N}(t)-\tilde{F}_{1, T}^{s, N}(t),
\end{aligned}
$$

where $h_{1}^{s}=h_{1}(1380)$. Recent studies in lattice QCD show that the vector [102] and tensor [49] strangeness content of the nucleon is tiny, leading to the estimates in Table I. In fact, (34) even predicts that $\kappa_{T}^{s, N} \equiv 0$. For strangeness, the uncertainties in the vector matrix elements need to be included, we use $\mu^{s}=0.006(4),\left\langle r_{E, S}^{2}\right\rangle^{N}=0.0012(9) \mathrm{fm}^{2}$, $\left\langle r_{M, s}^{2}\right\rangle^{N}=0.0014(27) \mathrm{fm}^{2}$ [102]. The matrix elements of the heavy quarks $c, b, t$ can be addressed using the heavy-quark expansion [103].

In conclusion, our main results in (33) and Table I summarize the present status of meson and nucleon form factors of the antisymmetric tensor current, using all available information from lattice QCD, analyticity, 
unitarity, and pole dominance. While, in general, the momentum dependence of the form factors can be reconstructed quite accurately, for the normalizations, input from lattice QCD is key, and the dominant uncertainties in the normalization of the induced nucleon form factors precisely reflect the fact that the sum rules for the normalization exhibit rather slow convergence. However, the combination of all available information allows us to provide reliable results both for the normalizations and momentum dependence, including form factors that previously could only be estimated using hadronic models [104-107]. The method developed here could be extended to hyperon tensor form factors and thus improve the matrix elements required in the search for nonstandard hyperon decays [108].

We thank Gilberto Colangelo, Aurore Courtoy, and Ulf-G. Meißner for comments on the manuscript. Financial support by the DOE (Grants No. DE-FG02-00ER41132 and No. DESC0009919), the DFG and NSFC through funds provided to the Sino-German CRC 110 "Symmetries and the Emergence of Structure in QCD" (DFG Grant No. TRR110 and NSFC Grant No. 11621131001), and the Swiss National Science Foundation (Projects No. PZ00P2_174228 and No. P300P2_167751) is gratefully acknowledged.

[1] S. Weinberg, Phys. Rev. Lett. 43, 1566 (1979).

[2] W. Buchmüller and D. Wyler, Nucl. Phys. B268, 621 (1986).

[3] B. Grzadkowski, M. Iskrzyński, M. Misiak, and J. Rosiek, J. High Energy Phys. 10 (2010) 085.

[4] M. Gell-Mann and M. Lévy, Nuovo Cimento 16, 705 (1960).

[5] M. Gell-Mann, Physics 1, 63 (1964).

[6] S. L. Glashow and S. Weinberg, Phys. Rev. Lett. 20, 224 (1968).

[7] M. González-Alonso and J. Martin Camalich, Phys. Rev. Lett. 112, 042501 (2014).

[8] S. Weinberg, Physica (Amsterdam) 96A, 327 (1979).

[9] J. Gasser and H. Leutwyler, Ann. Phys. (N.Y.) 158, 142 (1984).

[10] J. Gasser and H. Leutwyler, Nucl. Phys. B250, 465 (1985).

[11] C. G. Callan and S. B. Treiman, Phys. Rev. Lett. 16, 153 (1966).

[12] S. Weinberg, Phys. Rev. Lett. 17, 336 (1966); 18, 1178(E) (1967).

[13] R. F. Dashen and M. Weinstein, Phys. Rev. Lett. 22, 1337 (1969).

[14] V. Bernard, M. Oertel, E. Passemar, and J. Stern, Phys. Lett. B 638, 480 (2006).

[15] G. Abbas, B. Ananthanarayan, I. Caprini, I. Sentitemsu Imsong, and S. Ramanan, Eur. Phys. J. A 44, 175 (2010).

[16] G. Colangelo, E. Passemar, and P. Stoffer, Eur. Phys. J. C 75, 172 (2015).

[17] T. P. Cheng and R. F. Dashen, Phys. Rev. Lett. 26, 594 (1971).

[18] L. S. Brown, W. J. Pardee, and R. D. Peccei, Phys. Rev. D 4, 2801 (1971).
[19] J. Gasser, H. Leutwyler, and M. E. Sainio, Phys. Lett. B 253, 252 (1991).

[20] M. Hoferichter, J. Ruiz de Elvira, B. Kubis, and U.-G. Meißner, Phys. Rev. Lett. 115, 092301 (2015).

[21] O. Catà and V. Mateu, J. High Energy Phys. 09 (2007) 078.

[22] M. Diehl, Phys. Rep. 388, 41 (2003).

[23] A. Courtoy, S. Baeßler, M. González-Alonso, and S. Liuti, Phys. Rev. Lett. 115, 162001 (2015).

[24] Z. B. Kang, A. Prokudin, P. Sun, and F. Yuan, Phys. Rev. D 93, 014009 (2016).

[25] Z. Ye, N. Sato, K. Allada, T. Liu, J.-P. Chen, H. Gao, Z.-B. Kang, A. Prokudin, P. Sun, and F. Yuan, Phys. Lett. B 767, 91 (2017).

[26] H. W. Lin, W. Melnitchouk, A. Prokudin, N. Sato, and H. Shows, Phys. Rev. Lett. 120, 152502 (2018).

[27] M. Radici and A. Bacchetta, Phys. Rev. Lett. 120, 192001 (2018).

[28] G. Colangelo, G. Isidori, and J. Portolés, Phys. Lett. B 470, 134 (1999).

[29] B. Sciascia et al. (FlaviaNet Working Group), Nucl. Phys. B, Proc. Suppl. 181-182, 83 (2008); arXiv:0801.1817.

[30] H. Z. Devi, L. Dhargyal, and N. Sinha, Phys. Rev. D 90, 013016 (2014).

[31] V. Cirigliano, A. Crivellin, and M. Hoferichter, Phys. Rev. Lett. 120, 141803 (2018).

[32] J. A. Miranda and P. Roig, J. High Energy Phys. 11 (2018) 038.

[33] J. Goodman, M. Ibe, A. Rajaraman, W. Shepherd, T. M. P. Tait, and H. B. Yu, Phys. Rev. D 82, 116010 (2010).

[34] M. Hoferichter, P. Klos, and A. Schwenk, Phys. Lett. B 746, 410 (2015).

[35] M. Hoferichter, P. Klos, J. Menéndez, and A. Schwenk, Phys. Rev. D 94, 063505 (2016).

[36] F. Bishara, J. Brod, B. Grinstein, and J. Zupan, J. High Energy Phys. 11 (2017) 059.

[37] M. Hoferichter, P. Klos, J. Menéndez, and A. Schwenk, arXiv:1812.05617 [Phys. Rev. D (to be published)].

[38] V. Cirigliano, R. Kitano, Y. Okada, and P. Tuzon, Phys. Rev. D 80, 013002 (2009).

[39] A. Crivellin, M. Hoferichter, and M. Procura, Phys. Rev. D 89, 093024 (2014).

[40] A. Crivellin, S. Davidson, G. M. Pruna, and A. Signer, J. High Energy Phys. 05 (2017) 117.

[41] V. Cirigliano, S. Davidson, and Y. Kuno, Phys. Lett. B 771, 242 (2017).

[42] M. Pospelov and A. Ritz, Ann. Phys. (Amsterdam) 318, 119 (2005).

[43] J. Engel, M. J. Ramsey-Musolf, and U. van Kolck, Prog. Part. Nucl. Phys. 71, 21 (2013).

[44] D. Becirevic, V. Lubicz, G. Martinelli, and F. Mescia (SPQcdR Collaboration), Phys. Lett. B 501, 98 (2001).

[45] I. Baum, V. Lubicz, G. Martinelli, L. Orifici, and S. Simula, Phys. Rev. D 84, 074503 (2011).

[46] T. Bhattacharya, V. Cirigliano, R. Gupta, H. W. Lin, and B. Yoon, Phys. Rev. Lett. 115, 212002 (2015).

[47] C. Alexandrou et al., Phys. Rev. D 95, 114514 (2017); 96, 099906(E) (2017).

[48] N. Yamanaka, S. Hashimoto, T. Kaneko, and H. Ohki (JLQCD Collaboration), Phys. Rev. D 98, 054516 (2018). 
[49] R. Gupta, B. Yoon, T. Bhattacharya, V. Cirigliano, Y. C. Jang, and H. W. Lin, Phys. Rev. D 98, 091501 (2018).

[50] G. Ecker, J. Gasser, A. Pich, and E. de Rafael, Nucl. Phys. B321, 311 (1989).

[51] G. Ecker, J. Gasser, H. Leutwyler, A. Pich, and E. de Rafael, Phys. Lett. B 223, 425 (1989).

[52] W. R. Frazer and J. R. Fulco, Phys. Rev. 117, 1603 (1960).

[53] W. R. Frazer and J. R. Fulco, Phys. Rev. 117, 1609 (1960).

[54] G. Höhler, E. Pietarinen, I. Sabba Stefanescu, F. Borkowski, G. G. Simon, V. H. Walther, and R. D. Wendling, Nucl. Phys. B114, 505 (1976).

[55] P. Mergell, U.-G. Meißner, and D. Drechsel, Nucl. Phys. A596, 367 (1996).

[56] M. A. Belushkin, H.-W. Hammer, and U.-G. Meißner, Phys. Rev. C 75, 035202 (2007).

[57] I. T. Lorenz, U.-G. Meißner, H.-W. Hammer, and Y.-B. Dong, Phys. Rev. D 91, 014023 (2015).

[58] L. P. Gamberg and G. R. Goldstein, Phys. Rev. Lett. 87, 242001 (2001).

[59] K. M. Watson, Phys. Rev. 95, 228 (1954).

[60] R. Omnès, Nuovo Cimento 8, 316 (1958).

[61] J. F. de Trocóniz and F. J. Ynduráin, Phys. Rev. D 65, 093001 (2002).

[62] H. Leutwyler, arXiv:hep-ph/0212324.

[63] G. Colangelo, Nucl. Phys. B, Proc. Suppl. 131, 185 (2004).

[64] J. F. de Trocóniz and F. J. Ynduráin, Phys. Rev. D 71, 073008 (2005).

[65] C. Hanhart, Phys. Lett. B 715, 170 (2012).

[66] B. Ananthanarayan, I. Caprini, D. Das, and I. Sentitemsu Imsong, Phys. Rev. D 89, 036007 (2014).

[67] B. Ananthanarayan, I. Caprini, D. Das, and I. Sentitemsu Imsong, Phys. Rev. D 93, 116007 (2016).

[68] M. Hoferichter, B. Kubis, J. Ruiz de Elvira, H.-W. Hammer, and U.-G. Meißner, Eur. Phys. J. A 52, 331 (2016).

[69] C. Hanhart, S. Holz, B. Kubis, A. Kupść, A. Wirzba, and C. W. Xiao, Eur. Phys. J. C 77, 98 (2017); 78, 450(E) (2018).

[70] G. Colangelo, M. Hoferichter, and P. Stoffer, J. High Energy Phys. 02 (2019) 006.

[71] M. Diehl, A. Manashov, and A. Schäfer, Phys. Lett. B 622, 69 (2005).

[72] G. P. Lepage and S. J. Brodsky, Phys. Rev. D 22, 2157 (1980).

[73] W. Dekens, E. E. Jenkins, A. V. Manohar, and P. Stoffer, J. High Energy Phys. 01 (2019) 088.

[74] M. Tanabashi et al. (Particle Data Group), Phys. Rev. D 98, 030001 (2018).

[75] G. Colangelo, J. Gasser, and H. Leutwyler, Nucl. Phys. B603, 125 (2001).

[76] R. García-Martín, R. Kamiński, J. R. Peláez, and J. Ruiz de Elvira, Phys. Rev. Lett. 107, 072001 (2011).

[77] M. Hoferichter, B. Kubis, and M. Zanke, Phys. Rev. D 96, 114016 (2017).

[78] M. Hoferichter, J. Ruiz de Elvira, B. Kubis, and U.-G. Meißner, Phys. Rep. 625, 1 (2016).

[79] P. Federbush, M. L. Goldberger, and S. B. Treiman, Phys. Rev. 112, 642 (1958).
[80] S. Weinberg, Phys. Rev. 112, 1375 (1958).

[81] S. L. Adler, E. W. Colglazier, Jr., J. B. Healy, I. Karliner, J. Lieberman, Y. J. Ng, and H. S. Tsao, Phys. Rev. D 11, 3309 (1975).

[82] M. Göckeler, Ph. Hagler, R. Horsley, Y. Nakamura, D. Pleiter, P. E. L. Rakow, A. Schäfer, G. Schierholz, H. Stuben, and J. M. Zanotti (QCDSF and UKQCD Collaborations), Phys. Rev. Lett. 98, 222001 (2007).

[83] B. Kubis and R. Lewis, Phys. Rev. C 74, 015204 (2006).

[84] V. M. Braun et al., J. High Energy Phys. 04 (2017) 082 .

[85] O. Catà and V. Mateu, Phys. Rev. D 77, 116009 (2008).

[86] V. M. Braun, T. Burch, C. Gattringer, M. Göckeler, G. Lacagnina, S. Schaefer, and A. Schäfer, Phys. Rev. D 68, 054501 (2003).

[87] C. Allton et al. (RBC and UKQCD Collaborations), Phys. Rev. D 78, 114509 (2008).

[88] K. Jansen, C. McNeile, C. Michael, and C. Urbach (ETM Collaboration), Phys. Rev. D 80, 054510 (2009).

[89] O. Catà and V. Mateu, Nucl. Phys. B831, 204 (2010).

[90] E. L. Lomon and S. Pacetti, Phys. Rev. D 85, 113004 (2012); 86, 039901(E) (2012).

[91] B. Moussallam, Eur. Phys. J. C 73, 2539 (2013).

[92] M. Hoferichter, B. Kubis, S. Leupold, F. Niecknig, and S. P. Schneider, Eur. Phys. J. C 74, 3180 (2014).

[93] M. Hoferichter, B.-L. Hoid, B. Kubis, S. Leupold, and S. P. Schneider, J. High Energy Phys. 10 (2018) 141.

[94] G. Ecker and C. Zauner, Eur. Phys. J. C 52, 315 (2007).

[95] V. Bernard, N. Kaiser, and U.-G. Meißner, Nucl. Phys. A611, 429 (1996).

[96] M. Göckeler, Ph. Hägler, R. Horsley, D. Pleiter, P. E. L. Rakow, A. Schäfer, G. Schierholz, and J. M. Zanotti (QCDSF and UKQCD Collaborations), Phys. Lett. B 627, 113 (2005).

[97] L. Koester, W. Waschkowski, L. V. Mitsyna, G. S. Samosvat, P. Prokofjevs, and J. Tambergs, Phys. Rev. C 51, 3363 (1995).

[98] S. Kopecky, J. A. Harvey, N. W. Hill, M. Krenn, M. Pernicka, P. Riehs, and S. Steiner, Phys. Rev. C 56, 2229 (1997).

[99] R. Pohl et al., Nature (London) 466, 213 (2010).

[100] A. Antognini et al., Science 339, 417 (2013).

[101] Z. Epstein, G. Paz, and J. Roy, Phys. Rev. D 90, 074027 (2014).

[102] C. Alexandrou, M. Constantinou, K. Hadjiyiannakou, K. Jansen, C. Kallidonis, G. Koutsou, and A. Vaquero AvilésCasco, Phys. Rev. D 97, 094504 (2018).

[103] M. V. Polyakov and J. Sieverding, J. High Energy Phys. 01 (2016) 092.

[104] B. Pasquini, M. Pincetti, and S. Boffi, Phys. Rev. D 72, 094029 (2005).

[105] T. Ledwig, A. Silva, and H.-C. Kim, Phys. Rev. D 82, 054014 (2010).

[106] G. Erkol and A. Ozpineci, Phys. Lett. B 704, 551 (2011).

[107] T. Ledwig and H.-C. Kim, Phys. Rev. D 85, 034041 (2012).

[108] H. M. Chang, M. González-Alonso, and J. Martin Camalich, Phys. Rev. Lett. 114, 161802 (2015). 